\title{
A New Electronic Instrumentation Approach for the Acquisition of Microscopic Blood Smear Images for the Automatic Diagnosis of Anemia
}

\author{
Jerome Nango Alico', Sié Ouattara ${ }^{1,2}$, Alain Clement ${ }^{3}$ \\ ${ }^{1}$ Laboratoire des Sciences et Technologies de la Communication et de l'Information (LSTCI), Yamoussoukro, Cote D'Ivoire \\ ${ }^{2}$ Institut National Polytechnique Houphouët Boigny (INPHB), Yamoussoukro, Cote D’Ivoire \\ ${ }^{3}$ Institut Universitaire de Technologie d'Angers (IUT), Angers, France \\ Email: sie_ouat@yahoo.fr, sie.ouattara@inphb.ci
}

How to cite this paper: Alico, J.N., Ouattara, S. and Clement, A. (2020) A New Electronic Instrumentation Approach for the Acquisition of Microscopic Blood Smear Images for the Automatic Diagnosis of Anemia. Advances in Bioscience and Biotechnology, 11, 237-255.

https://doi.org/10.4236/abb.2020.116018

Received: April 28, 2020

Accepted: May 31, 2020

Published: June 3, 2020

Copyright $\odot 2020$ by author(s) and Scientific Research Publishing Inc. This work is licensed under the Creative Commons Attribution International License (CC BY 4.0).

http://creativecommons.org/licenses/by/4.0/

\begin{abstract}
Anemia is a blood abnormality that affects the quantity and quality of red blood cells in the human body. This sometimes banal sign spares no continent and no social stratum. This anomaly is generally appreciated through biological analyzes of patients' blood. These analyzes, which boil down to the knowledge of hemato-metric constants, cannot by themselves allow the characterization of certain forms of anemia in the sense that most anemia are related to the morphology and color of red blood cells. Our work in this paper is to perform blood smears on patients and perform a morphological and colorimetric analysis of red blood cells on these smears. This approach allowed us to highlight on each erythrocyte morphological and colorimetric descriptors to accurately identify the types of anemia by image processing methods. This identification is performed in an automated environment to allow pathologists to respond quickly to anemia-related emergencies and also improve the treatment to be conducted. This automation required the implementation of a new approach to electronic instrumentation and the acquisition of microscopic blood smear images for the automatic and rapid diagnosis of anemia.
\end{abstract}

\section{Keywords}

Anemia, Instrumentation, Acquisition, Automated, Blood Smears, Red Blood Cells, Morphological Descriptors, Colorimetric Descriptors, Image

Processing, Diagnosis

\section{Introduction}

With 1.62 billion people diagnosed worldwide, according to the database of the 
World Health Organization (WHO), anemia is a major public health problem [1]. It touches every continent without exception. According to the report of the World Health Organization, globally the prevalence is $24.8 \%$ of the world population with a predominance of preschool children (47.4\%) and a regression is observed in men (12.7\%) [1]. But a high prevalence is observed in South Asia, Central Africa and West Africa. In Africa, for example, this prevalence is $62.3 \%$ among children aged 0 to 5 years [2]. Anemia threatens human existence because it affects about half a billion women of childbearing age in the world: In 2011, 29\% (496 million) of non-pregnant women and 38\% (32.4 million) of pregnant women aged 15 to 49 years were anemic [3]. Anemia spares no social layer in the world. This condition, which affects the number and/or quality of red blood cells (lowering of hemoglobin ( $\mathrm{Hb}$ ) levels in human blood) comes in many forms with a variety of causes.

Their characterization becomes paramount in that it can influence decision-making via diagnosis.

Automata already exist to perform the blood count which is an automated examination to assess certain types of anemia. Indeed, they make it possible to obtain information on the number and size of red blood cells [4]. But these two parameters do not provide enough information for a true characterization of anemia. Anemia can be categorized through the morphology and color of red blood cells (erythrocytes) observed on microscopic images obtained from blood smears performed on patients. Microscopic observation of blood smear images shows that there are different types of abnormalities related to the morphology and color of red blood cells [5]. Indeed, the combination of these two parameters will allow the detection of the type of anemia to clearly guide pathologists. Because depending on the shape or the color of the red blood cells we can affirm that it is about such a type of anemia and not of such another as the different images show it below (of Figure 7 in Figure 10) in the result section.

In addition, medical personnel sometimes use manual methods to characterize anemia. This detection technique is rudimentary, difficult and very subjective (observation of the coloring of palms, conjunctivae and nails ...). For effective management of patients and to overcome the shortcomings of the various methods mentioned above, we propose a new instrumentation approach for the automatic and rapid diagnosis of anemia.

The work we are conducting reveals morphological and colorimetric descriptors that are discriminant extracted on each form of red blood cells. In this perspective, work has been done in the literature. Indeed, according to Chantal Fossat et al. in 2006 [6] the automated evaluators of schizocytes (fragments of red blood cells) present difficulties (limits) concerning the formal identification of these. The approach discussed in this paper may rule out some diagnostic assumptions or may highlight morphological abnormalities of erythrocytes for better diagnostic accuracy [7]. The detection of schizocytes (fragment of red blood cells) fortuitously on the blood smear is a key indicator for the diagnosis of thrombotic microangiopathic anemia, which represents a proven medical 
emergency [8]. But the schizocyte search method still remains manual. In 2017, Hany A. Elsalamony, for the identification of sickle cell red blood cells, proposed a method based on the signature of their geometric form [9] [10]. Frejilichowski, 2011 developed a method for identifying abnormal red blood cells using a polar-Fourier grayscale descriptor on May-Grumwald-Giemsa stained blood smear images [11]. The compactness melting and the moment invariant of $\mathrm{HU}$ is the method used by R. Tomaria et al. 2014 to identify erythrocytes [12]. Das et al. in 2012 for the detection of certain forms of red blood cells used only certain morphological descriptors. Multi-class logistic regression is chosen because of its classification accuracy of normal and abnormal erythrocytes [13].

The implementation of an automated system according to Das et al. in 2012 [13] requires an instrumentation capable of performing a treatment of microscopic images resulting from blood smears. Then, the automatic extraction and the calculation of the different erythrocyte discriminating parameters morphologically and colorimetrically are carried out. The next article will aim to set up a method of classification or automatic recognition of the types of anemia suffered by a patient.

\section{Materials and Methods}

\subsection{Materials}

The characterization of the morphological and colorimetric of the red blood cells requires the production of blood smears on the various samples taken from healthy patients or from anemic patients. This realization requires the equipment of Figure 3 below. The tools used for the realization of this study are apart from Figure 3, a computer, a microscope, a camera and software allowing us to capture microscopic images. This work will produce data in the field and data collected following the analysis of the images obtained. the data in the field will be essentially composed of those obtained by the automated machines of the laboratories and the laboratory data are collected following the analysis of microscopic images of blood smears which are parameters discriminating each form or colorimetric aspect of the red blood cell as indicated the characteristics calculated and grouped in the tables below.

\subsubsection{Selection of Samples}

The laboratory data come from the samples of thirty (30) healthy people and one hundred and twenty (120) anemic people. We carried out five (05) blood smears per patient and, after the visual analysis of the practitioners we chose two (02) blood smears respecting the recommendations of the World Health Organization (WHO) [14]. Is performed randomly and comes from the health services of the city of Yamoussoukro including the pediatrics, medicine, surgery and gynecology services of the regional hospital center (CHR) and also of the Transfusion Center (CTB) of said city. In order to respect the principles of our study, we took patients whose anemic state was proven or not following the field data provided by laboratory automata. 


\subsubsection{Perform a Blood Smear}

The blood smear consists of spreading a drop of blood evenly on an object slide, so as to obtain a single layer on half of the surface of the slide [14]. After fixing and coloring, the morphological and colorimetric study of the figured elements of the blood can be carried out. To stick to our main objective, only red blood cells will interest us in this study. The different blood smears will each carry an identifier which corresponds to the patient from whom the sample is taken.

\subsubsection{How to Prepare a Smear}

- Mark the slide.

- Apply a drop of venous blood $1 \mathrm{~cm}$ from the tip of the object's blade, placed on a hard, horizontal plane.

- Hold the first slide with one hand and tilt the second slide $45^{\circ}$ just in front of the drop.

- Gradually put the slide in contact with the drop of blood.

- Let the blood flow along the stop of the second blade and before it reaches the edges, in a fast, uniform and continuous movement, pull the blood forward.

- Dry quickly while shaking (Figure 1) [15].

\subsubsection{Staining of a Blood Smear}

There are two staining strategies with Giemsa: the fast method (10\% dye) and the slow method ( $3 \%$ dye). The first is used in busy clinics and laboratories where speed of diagnosis is an essential element in the management of patients. The slow method is used to stain more slides, as is the case in epidemiological surveys. Given the large influx in the laboratories and the expected result we preferred the staining method to $10 \%$.

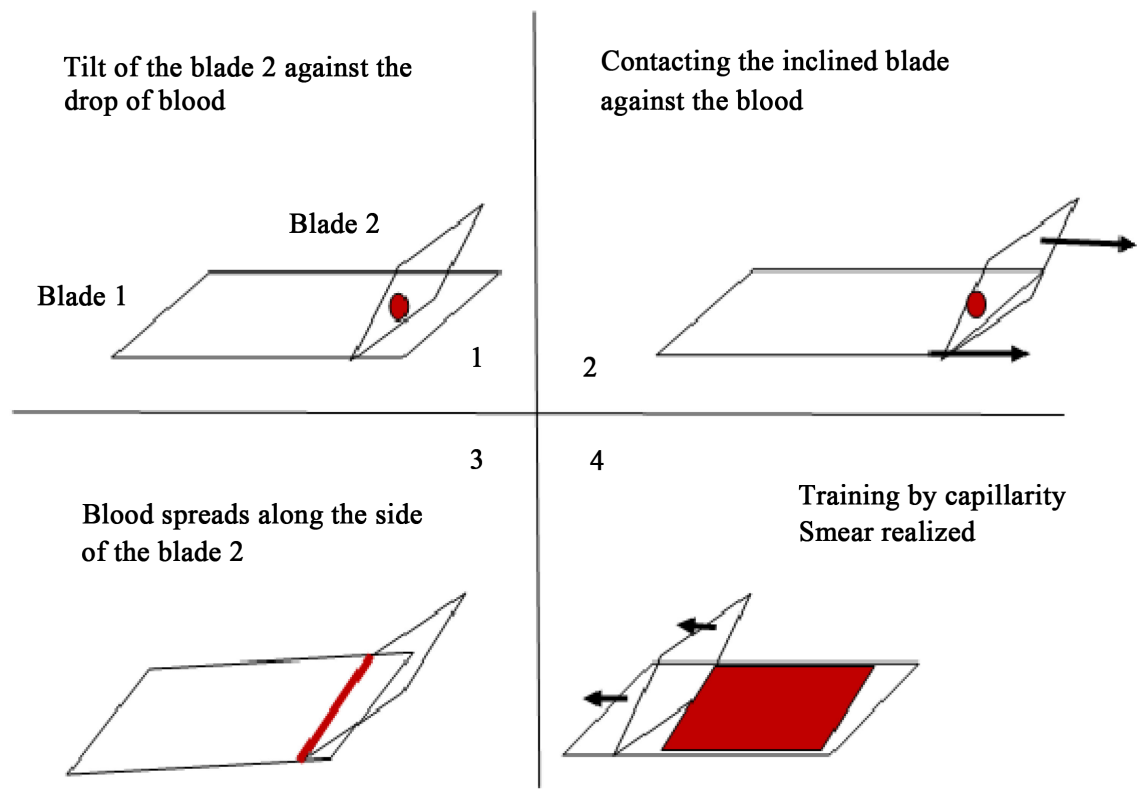

Figure 1. Demonstration of the blood smear technique 
There are usually two techniques for staining blood smears (by overlay and bath). We chose the bath staining technique instead of the stain technique. This technique has the advantage of staining several blood smears at a time, which is what guided our choice.

The process of bath staining of blood smears consists in following in chronological order the following steps:

- Preparation of three jars, the first containing a pure solution of May-Grunewald. the second containing a buffer solution and finally the third jar a solution of Giemsa diluted 1/10.

- Dive into the first jar of blood smears for five minutes (5 min).

- Transfer of blood smears into the jar containing the buffer solution.

- Removal of the smears from the buffer solution and soaking for 15 minutes in the jar containing Giemsa diluted 1/10 with distilled water pH 7.2.

- Rinse thoroughly 2 - 3 times each slide under running water.

- Exposure of the blades to the open air for drying.

- Wait for complete drying before the acquisition (Figure 2 and Figure 3).

\subsubsection{Acquisition of Microscopic Images}

The microscope and the Moticam 2.0 camera are mounted to make it a single entity. The block thus obtained is connected with a USB cable to the microcomputer on which the Moticam 3.0 software is installed, see Figure 4 below. This set will promote the automatic acquisition of microscopic color images of the blood smear. These images will be scanned and stored on the microcomputer with the installed software.

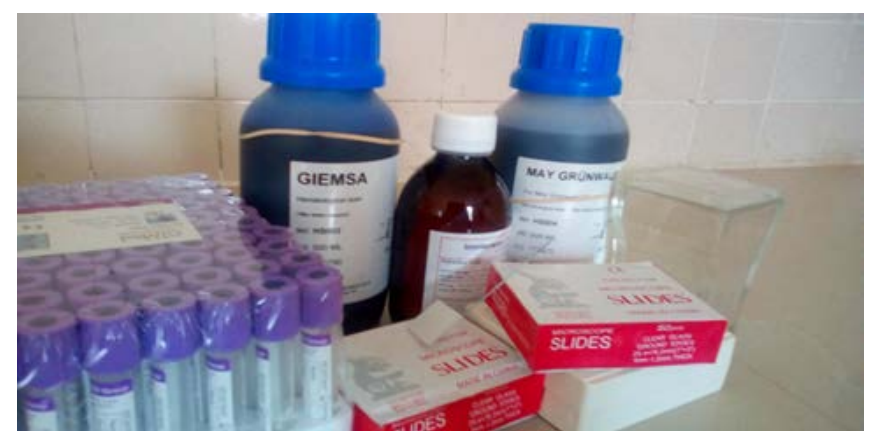

Figure 2. Example of non-colored blood smear.

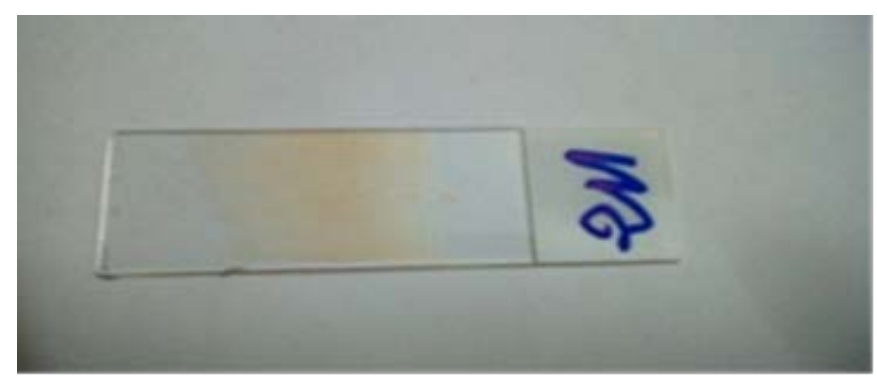

Figure 3. Colored smears for acquisition images.

https://pixers.fr/tableaux-sur-toile/frottis-sanguin-FO34994755. 


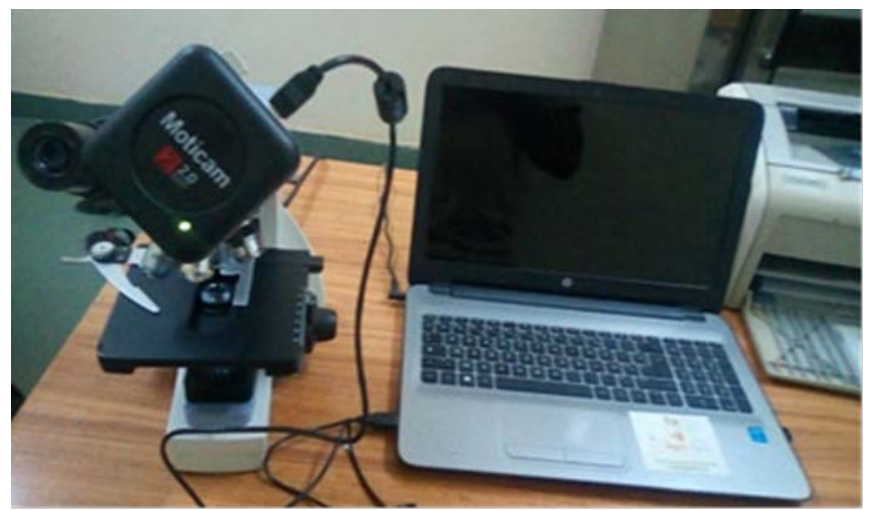

Figure 4. Image acquisition device.

\section{1) The Characteristics of the Equipment}

Capturing microscopic images for the proper conduct of our work required the acquisition of the following equipment: a microscope, a camera and a laptop. The characteristics of the camera and the microscope are grouped respectively in Table 1 and Table 2 below.

\section{1) The Characteristics of the Computer}

The entire device runs on an hp laptop with the following features:

- Processor: Intel ${ }^{\varpi}$ Core $^{\mathrm{TM}}$ i3-5005U processor at $2.00 \mathrm{GHz}$ at $2.00 \mathrm{GHz}$.

- Operating system: Windows10 Professional.

- RAM: 4, 00 Go.

- System type: 64 -bit operating system, $\times 64$ processors.

\subsection{Methods}

The proposed method starts from the acquisition of microscopic color images of the selected blood smears. Then we treat and characterize the different morphological and colorimetric parameters of each form of red blood cell (Figure 5).

\section{Morphological and Colorimetric Characterization}

The morphological and colorimetric characterization of red blood cells requires the extraction of different discriminating descriptors for each form of red blood cells. Indeed, the recognition of red blood cell forms is based on measurable data extracted on red blood cells. These data or characteristics must be discriminating in order to achieve good identification [16]. In this section we will present for each red cell the appropriate descriptor.

In this approach we need to isolate the discriminating red blood cell by an image processing tool that has segmentation.

\section{1) Segmentation}

Segmentation is the partition of an image into several regions according to a well defined criterion and having pixels of the same characteristics. The main purpose of this treatment is to extract the information that must allow a precise identification of the object concerned [16]. In the literature several segmentation methods have been developed to identify blood cells [9] [13] [17] [18]. In 2015, 


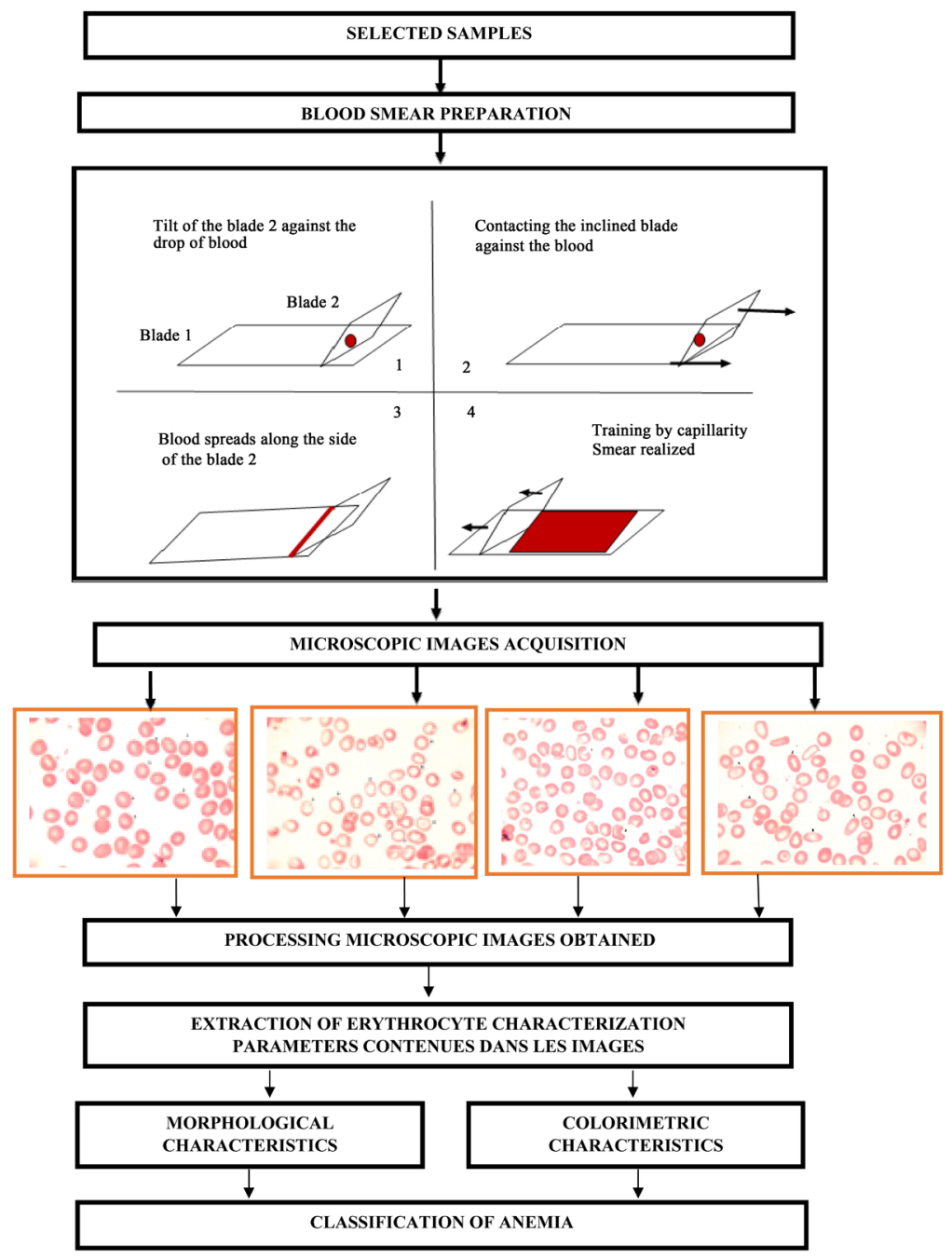

Figure 5. Flowchart of the proposed methodology.

Table 1. The characteristics of the camera.

\begin{tabular}{cc}
\hline Parameters values & Values \\
\hline Moticam & 2 \\
Sensor type & CMOS \\
Optical & $1 / 3^{\prime \prime}$ \\
Actives pixels & $1600 \times 1200$ \\
Pixel size & $3.2 \mu \mathrm{m} \times 3.2 \mu \mathrm{m}$ \\
Imaging Area & $5.12 \mathrm{~mm} \times 3.84 \mathrm{~mm}$ \\
Scan Mode & progressive \\
Shutter type & Rolling with Global reset \\
Operating temp & $-10^{\circ} \mathrm{C}$ to $60^{\circ} \mathrm{C}$ non condensing \\
Max. SNR & $43 \mathrm{~dB}$ \\
D/R & $61 \mathrm{~dB}$ \\
Interface & USB2.0 \\
Operating req. & Windows7 or higher, osx, Linux, $250 \mathrm{MB}$ free HD space \\
\hline
\end{tabular}


Table 2. Characteristics of microscope.

\begin{tabular}{cc}
\hline Parameters & Values \\
\hline Mechanism Tube length & $160 \mathrm{~mm}$ \\
Viewing Head & Compensation Free Trinocular Head, inclined at 30, \\
interpupillar distance $55-75 \mathrm{~mm}$ & Viewfield line $18 \mathrm{~mm}$ \\
Eyepiece & Forward Quadruple Nosepiece \\
Nosepiece & Achromatique: $4 \times, 10 \times, 40 \times, 100 \times$ \\
Object & Coaxial coarse and Fine Focusing \\
& System, sensitivity and Graduation of Fine Focus: $0.002 \mathrm{~mm}$. \\
Focus System & coarse \& fine focus range: $23 \mathrm{~mm}$ \\
& Abel, NA $=1.25$ \\
Condenser & Double layer mechanical stage, area: $140 \times 140 \mathrm{~mm}$, \\
Stage & movement range: $75 \times 50 \mathrm{~mm}$ \\
Lamp-House & Halogenlamp $6 \mathrm{~V} 20 \mathrm{~W}$ \\
&
\end{tabular}

the study by P. Shivhare et al., listed three main methods of segmentation [16]:

- Edge-based segmentation

- Segmentation based on the region

- Segmentation based on thresholding

\section{a) Edge-based segmentation}

This method is based on the abrupt change in pixel luminance that determines the contour. It makes it possible to mark a border or a real transition zone between the regions (the objects) and the background of the image.

\section{b) Segmentation based on the region}

The region in an image is the grouping of a number of pixels having similar values. This method then makes it possible to group a number of homogeneous pixels or having common attributes [19].

\section{c) Segmentation based on thresholding}

Thresholding segments an image into two classes. The intensity of each pixel is compared to the defined threshold and that pixel by pixel. When this intensity is below the threshold, the pixel takes the value 0 and the value 1 otherwise. This thresholding operation applied to the entire image leads to binarization of the image [20].

\section{2) Morphological descriptors}

Healthy red blood cells have a uniformly rounded and undeformed shape whereas those that are anemic have various forms that are often specific to the type of anemia. This morphological deformation can be characterized by shape descriptors:

a) Area of the red blood cells

The surface of the red cell or area is the set of pixels covering the segmented image.

$$
\text { area }=\sum_{x} \sum_{y} f(x, y)
$$


$f(x, y)$ is the pixel whose position is represented by the pair of $x$ and $y$ coordinates in the binarized image. It is 1 when the pixel is in the segmented region and 0 otherwise.

\section{b) Perimeter of the red blood cells $(P)$}

The perimeter is the sum of the pixels on the edge of the segmented image. To calculate it we will use the eight-connexity method (Table 3).

We go through the whole contour. When a pixel has a connectivity lower than 8 this pixel belongs to the outline.

$$
P=\sum_{x} \sum_{y} f(x, y)
$$

\section{c) Compactness $(C)$}

The compactness of the region is the ratio between the area of this region and the perimeter. It measures the regularity of the surface of the region

$$
C=\frac{4 \times \pi \times \text { area }}{p^{2}}
$$

\section{d) Eccentricity (e)}

Eccentricity describes the degree of elongation of a red blood cell.

$$
e=\left(1-\frac{b^{2}}{a^{2}}\right)^{1 / 2}
$$

The variable $b$ represents the minor axis and the variable a represents the major axis. The value of eccentricity varies between 0 and 1 for red blood cell forms. When $e=0$ the object is practically a circle, when $e<1$ the object is lengthened.

\section{e) Convex set}

Let two distinct pixels $x$ and $y$ belong to the same red cell $(\mathcal{E})$. The number of pixels separating them describes a segment $[x, y]$. If the entire segment belongs to the cell whatever $x$ and $y$ then the set is convex. This method allows us to clearly characterize certain erythrocytes.

$$
\{(\mathcal{E}) \text { convex if } \forall x, y \in(\mathcal{E}), t x+(1-t) y, t \in[0,1]\}
$$

The segment $[\mathrm{x}, \mathrm{y}]$ is defined as follows:

$$
\{(x, y) / t x+(1-t) y, t \in[0,1]\}
$$

$t$ is the variable describes the segment $[x, y]$

\section{3) Color Descriptors}

\section{a) Average pixel intensity}

An image is a set of pixels, each of which has a value that defines its intensity. So the average intensity of this region can be known. We have therefore from the

Table 3. The eight-connexity neighbours of the pixel $(x, y)$.

$\begin{array}{ccc}(x-1, y-1) & (x, y-1) & (x+1, y-1) \\ (x-1, y) & (x, y) & (x+1, y) \\ (x-1, y+1) & (x, y+1) & (x+1, y+1)\end{array}$


algorithm below calculated the average intensity of the pixels for each region. The algorithm is designed under MATLAB 2016a. Its principle is defined hereafter:

$$
\begin{aligned}
& \text { b = view image (filename); } \\
& \text { im = read image (filename); } \\
& \text { im2 = converting colors to grayscale (im); } \\
& \text { h = selection region; } \\
& \text { bw = conversion to binary image (h, b); } \\
& \text { view image (bw); } \\
& \text { Imoy = Mean color of object in white. }
\end{aligned}
$$

b) Entropy

Entropy is a statistical measure to obtain information. It becomes an important parameter to characterize an image.

$$
H(x)=\sum_{i=1}^{n} P_{i} \cdot \log _{2}\left(P_{i}\right)
$$

\section{c) Standard deviation $(\sigma)$}

This statistical tool is the gap between the intensity of each pixel to the average of the pixel intensity.

$$
\sigma=\sqrt{\frac{1}{n-1} \sum_{i=1}^{n}\left(x_{i}-\bar{x}\right)^{2}}
$$

\section{d) Percentage of colored area in red (PCol)}

The colored part of the red blood cell contains hemoglobin hence the red color of the cells.

$$
\text { PCol }=((\text { areag }- \text { areab }) / \text { areag }) * 100
$$

Areag: represents the number of pixels of the total area of the red blood cell, Areab: the number of pixels of the central white part of the erythrocyte,

PCol: represents the percentage of ocqupation of the colored area of the red blood cell.

e) Area of the white zone of the red blood cells

$$
\begin{gathered}
\text { areablc }=\text { areag }- \text { areacol } \\
\text { pblc }=(\text { areablc } / \text { areag }) \times 100
\end{gathered}
$$

Areablc: is the set of pixels in the central area of the red cell,

Areacol: the set of pixels in the colored area.

\section{Results and Discussions}

Examination of the blood smear makes it possible to carry out a morphological and colorimetric study of the various figured elements of the blood. For our work, only red blood cells interest us. On a normal smear, red blood cells have rounded shapes, without nuclei and the same color. Any modification of these parameters reflects a pathological condition as shown in Figures 8-10 below. Anemia is a consequence of these changes. 
In this section, we will present the results obtained during the acquisition and identification phase of red blood cells.

\subsection{Some Acquisitions of Blood Smear Images from Different Patients}

For each blood smear we made images of several optical fields of different patients. In order to obtain quality and exploitable images, we played on certain parameters of the camera. These are the following parameters (Figure 6):

- Color (Gain and brightness of RGB colors).

- Brightness.

- Resolution, exposure, contrast, gain.

- White balance, gamma.

We present in this section some images. Figure 7 below shows a normal smear image characterized by circular, coreless red blood cells with a slightly white area in the center. These different cells have approximately the same color and the same morphology. Such a smear shows the absence of anemia.

Anemia with hypochromic erythrocytes is anemia that is caused by iron deficiency in the body. It is also called iron deficiency anemia. This type of anemia is common in medicine. It is characterized by a pallor of red blood cells with the white central area more developed and it is found that the hemoglobin (red part) is deposited at the periphery of the red blood cells forming a ring as shown in Figure 8 below. It can also be observed in chronic hemorrhages (gastric ulcers, gastric cancer, hemorrhoids ...).

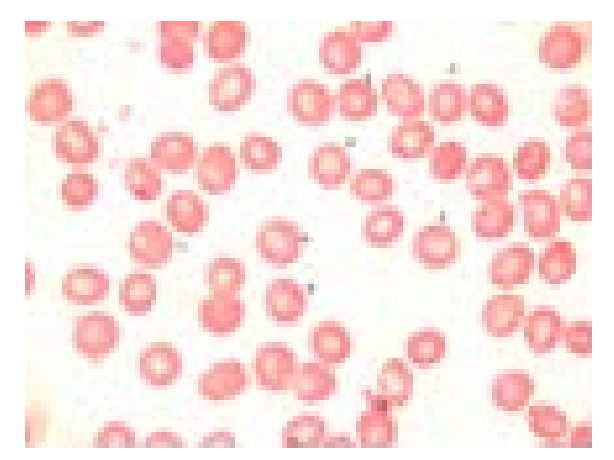

Figure 6. Smear image showing rounded red cells with the same red color.

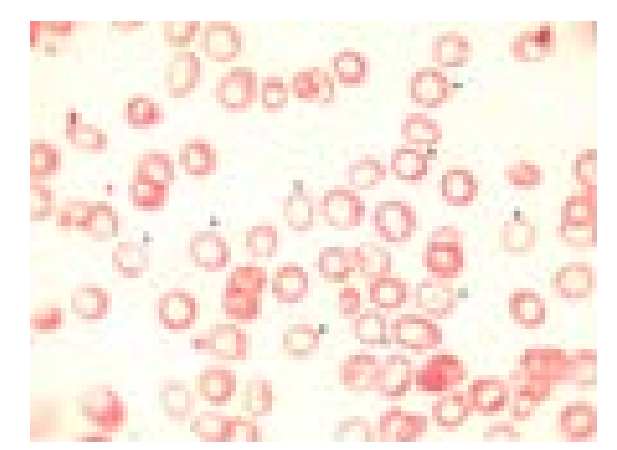

Figure 7. Image showing pale red blood cells, low in hemoglobin (hypochromia). 


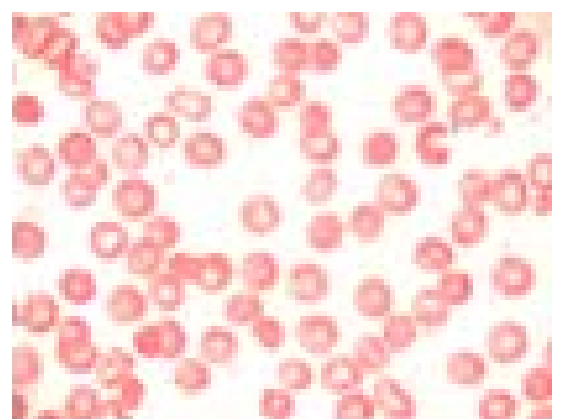

(a)

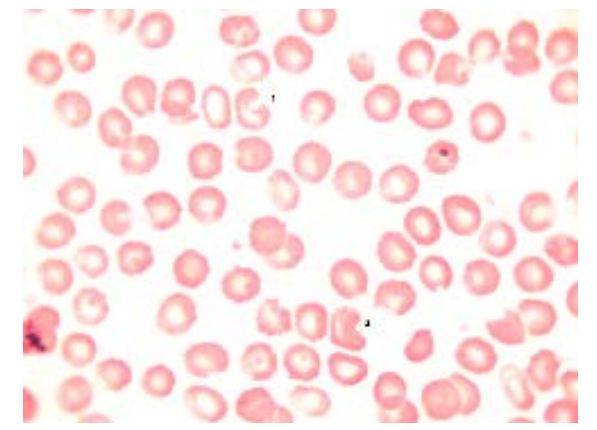

(b)

Figure 8. Numbers (1) and (2) of Figures (a) and (b) show sickle cells.

Figure 9 below shows deformed red blood cells in the shape of a sickle or banana. They are responsible for sickle cell disease, which is a disease of the hemoglobin (A and S) genes. The gene $A$ is said to be normal, whereas the $S$ gene is responsible for the deformation of the red blood cell skeleton in the shape of a sickle or banana. The detection of such a morphological abnormality is possible on the blood smear.

Figure 10 shows the image of a blood smear on which red blood cells numbered $1-6$ have elongated shapes with rounded ends: they are elliptocytes. This form of red blood cells may be present in the healthy subject (less than 1\%) but in the case of hereditary elliptocytosis the number of elliptocytes may vary from $10 \%$ to $90 \%$ of the red blood cells [21]. The optical images were obtained with the objective 100 times $(100 \times)$, which is equivalent to a magnification $(\mathrm{G})$ of 1000 (eyepiece: $10 \times$ and objective $100 \times$, i.e. $\mathrm{G}=10 \times 100$ ), allowing a morphological and colorimetric study of the Red blood cells.

\subsection{Images of Some Segmentation and Measurement of Morphological and Colorimetric Parameters on Red Blood Cells}

The characterization of a red blood cell requires both measured morphological and colorimetric data. The success of this action leads to the use of an image processing tool: segmentation. The advantage of this tool is to isolate the cell in order to extract information that can identify it accurately. Segmentation methods are numerous [16] as defined in Section 2.4.1. But to extract information from each of the red blood cells we propose a method of segmentation semi-supervised by the selection of the contours of each cell. We segment the isolated red blood cell three times, then record the different measurements and average them as shown in Tables 4-11. This method will result in isolation of the cell as shown in Figure 10(a) and Figure 10(b). We find that the images in Figure 10 highlight the cell in white on a black background. All the information to extract is in the white part that represents the segmented red blood cell. This information is obtained through algorithms that we developed and implemented under MATLAB 2016a. The results obtained on each erythrocyte or red blood cell are recorded in a table, some of which are presented in Tables 4-11 below. 
Table 4. Morphological parameters of healthy red blood cell.

\begin{tabular}{cccccc}
\hline cap269 & area & perimeter & compactness & eccentricity & pbinary \\
\hline H1 & 5559 & 274 & 0.93048 & 0.3400 & 0 \\
& 5557 & 270 & 0.95791 & 0.2910 & 0 \\
MH1 & 5466 & 273 & 0.92162 & 0.3212 & 0 \\
H2 & 5527.3333 & 272.3333 & 0.93667 & 0.3174 & 0 \\
& 5416 & 273 & 0.9132 & 0.2803 & 0 \\
& 5400 & 267 & 0.9519 & 0.3287 & 0 \\
MH2 & 5598 & 272 & 0.9508 & 0.1793 & 0 \\
H3 & 5471.3333 & 270.6667 & 0.9386 & 0.2628 & 0 \\
& 5727 & 278 & 0.9312 & 0.3823 & 0 \\
& 5800 & 279 & 0.9363 & 0.3162 & 0 \\
MH3 & 5717 & 274 & 0.9569 & 0.2847 & 0 \\
\hline
\end{tabular}

Table 5. Colorimetric parameters of healthy red blood cells.

\begin{tabular}{cccccccc}
\hline cap269 & intMoy & std & colMR & ColMG & ColMB & \%colored & \%white \\
\hline \multirow{2}{*}{ H1 } & 202 & 5.1568 & 255 & 224 & 220 & 94.2616 & 5.7384 \\
& 202 & 5.1547 & 255 & 224 & 220 & 94.3134 & 5.6865 \\
MH1 & 202 & 5.1153 & 255 & 224 & 220 & 94.1090 & 5.8910 \\
& 202 & 5.1423 & 255 & 224 & 220 & 94.2280 & 5.7720 \\
H2 & 204 & 5.7267 & 255 & 224 & 220 & 83.4564 & 16.5436 \\
& 204 & 5.7797 & 255 & 224 & 220 & 84.0741 & 15.9259 \\
MH2 & 204 & 5.8043 & 255 & 224 & 220 & 84.8875 & 15.1125 \\
& 204 & 5.7702 & 255 & 224 & 220 & 84.1393 & 15.8606 \\
H3 & 204 & 5.4969 & 255 & 224 & 220 & 90.3964 & 9.6036 \\
& 204 & 5.5139 & 255 & 224 & 220 & 90.1724 & 9.8276 \\
MH3 & 204 & 5.4615 & 255 & 224 & 220 & 89.9948 & 10.0052 \\
\hline
\end{tabular}

Table 6. Annulocyte morphological parameters.

\begin{tabular}{cccccc}
\hline Cap311 & area & perimeter & compactness & eccentricity & pbinary \\
\hline H1 & 4171 & 241 & 0.9024 & 0.4940 & 0 \\
& 4123 & 234 & 0.9462 & 0.5723 & 0 \\
MH1 & 4036 & 235 & 0.9184 & 0.3815 & 0 \\
& 4110 & 236.6667 & 0.9223 & 0.4826 & 0 \\
H2 & 3403 & 215 & 0.9251 & 0.4013 & 0 \\
& 3558 & 220 & 0.9238 & 0.4806 & 0 \\
MH2 & 3428 & 213 & 0.9495 & 0.4523 & 0 \\
& 3463 & 216 & 0.9328 & 0.4447 & 0 \\
H3 & 3975 & 229 & 0.9525 & 0.3500 & 0 \\
& 4294 & 240 & 0.9368 & 0.2797 & 0 \\
MH3 & 4097 & 234 & 0.9402 & 0.3008 & 0 \\
\hline
\end{tabular}


Table 7. Annulocyte colorimetric parameters.

\begin{tabular}{cccccccc}
\hline Cap311 & intMoy & std & colMR & ColMG & ColMB & \%colored & \%white \\
\hline \multirow{3}{*}{ H1 } & 228 & 18.2366 & 254 & 223 & 212 & 60.1295 & 39.8705 \\
& 228 & 18.4479 & 254 & 223 & 212 & 62.6728 & 37.3272 \\
MH1 & 228 & 18.4187 & 254 & 223 & 212 & 59.6878 & 40.3122 \\
& 228 & 18.3678 & 254 & 223 & 212 & 60.8300 & 39.1700 \\
H2 & 219 & 25.7382 & 254 & 223 & 212 & 62.8857 & 37.1143 \\
& 219 & 25.5280 & 254 & 223 & 212 & 62.4227 & 37.5773 \\
MH2 & 219 & 25.7352 & 254 & 223 & 212 & 62.3396 & 37.6604 \\
& 219 & 25.6671 & 254 & 223 & 212 & 62.5493 & 37.4507 \\
H3 & 219 & 24.9620 & 254 & 223 & 212 & 59.4214 & 40.5786 \\
& 219 & 24.4978 & 254 & 223 & 212 & 62.8319 & 37.1681 \\
MH3 & 219 & 24.7150 & 254 & 223 & 212 & 61.5572 & 38.4428 \\
\hline
\end{tabular}

Table 8. Morphological parameters sickle cell.

\begin{tabular}{cccccc}
\hline Cap227 & area & perimeter & compactness & eccentricity & Pbinary \\
\hline H1 & 2855 & 278 & 0.4642 & 0.8672 & 1 \\
& 2981 & 276 & 0.4918 & 0.8813 & 1 \\
MH1 & 2971 & 275 & 0.4937 & 0.8553 & 1 \\
& 2935.6667 & 276.3333 & 0.4832 & 0.8680 & 1 \\
H2 & 2208 & 207 & 0.6475 & 0.7560 & 1 \\
& 2098 & 201 & 0.6526 & 0.8140 & 1 \\
MH2 & 2183 & 207 & 0.6402 & 0.8329 & 1 \\
& 2163 & 205 & 0.6468 & 0.8010 & 1 \\
H3 & 3295 & 271 & 0.5638 & 0.8413 & 1 \\
& 3358 & 275 & 0.5580 & 0.8372 & 1 \\
MH3 & 3309 & 268 & 0.5790 & 0.8346 & 1 \\
\hline
\end{tabular}

Table 9. Sickle cell colorimetric parameters.

\begin{tabular}{cccccccc}
\hline Cap227 & intMoy & std & colMR & ColMG & ColMB & \%coloré & \%blanc \\
\hline \multirow{2}{*}{ H1 } & 175 & 16.8154 & 255 & 222 & 215 & 100 & ABS \\
& 175 & 18.3393 & 255 & 222 & 215 & 100 & ABS \\
MH1 & 175 & 18.1241 & 255 & 222 & 215 & 100 & ABS \\
& 175 & 17.7596 & 255 & 222 & 215 & 100 & ABS \\
H2 & 184 & 20.8689 & 253 & 214 & 204 & 100 & ABS \\
& 184 & 20.0974 & 253 & 214 & 204 & 100 & ABS \\
MH2 & 184 & 20.5583 & 253 & 214 & 204 & 100 & ABS \\
& 184 & 20.5082 & 253 & 214 & 204 & 100 & ABS \\
H3 & 197 & 13.2418 & 255 & 213 & 206 & 100 & ABS \\
& 197 & 14.3507 & 255 & 213 & 206 & 100 & ABS \\
MH3 & 197 & 13.8554 & 255 & 213 & 206 & 100 & ABS \\
\hline
\end{tabular}




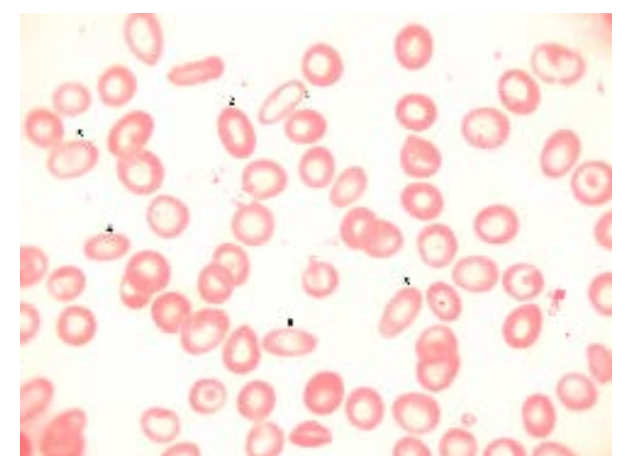

Figure 9. Smears with numbered red blood cells elliptical shapes called elliptocytes.

Table 10. Morphological parameters elliptocytes.

\begin{tabular}{cccccc}
\hline Cap229 & area & perimeter & compactness & eccentricity & Pbinary \\
\hline \multirow{2}{*}{ H1 } & 4013 & 251 & 0.8004 & 0.8727 & 0 \\
& 3923 & 245 & 0.8213 & 0.8738 & 0 \\
MH1 & 4029 & 251 & 0.8036 & 0.8781 & 0 \\
& 3988.33 & 249 & 0.8085 & 0.8749 & 0 \\
H2 & 4500 & 252 & 0.8905 & 0.7476 & 0 \\
& 4801 & 267 & 0.8463 & 0.7860 & 0 \\
MH2 & 4801 & 267 & 0.8463 & 0.7860 & 0 \\
& 4700.667 & 262 & 0.8610 & 0.7732 & 0 \\
H3 & 4272 & 256 & 0.8191 & 0.8513 & 0 \\
& 4210 & 259 & 0.7887 & 0.8739 & 0 \\
MH3 & 4211 & 253 & 0.8267 & 0.8522 & 0 \\
\hline
\end{tabular}

Table 11. Elliptocyte colorimetric parameters.

\begin{tabular}{cccccccc}
\hline Cap229 & intMoy & std & colMR & ColMG & ColMB & \%colored & \%white \\
\hline \multirow{2}{*}{ H1 } & 232 & 17.1237 & 255 & 233 & 228 & 68.9758 & 31.0242 \\
& 232 & 17.1677 & 255 & 233 & 228 & 67.0150 & 32.985 \\
MH1 & 232 & 17.0458 & 255 & 233 & 228 & 68.3544 & 31.645 \\
& 232 & 17.1122 & 255 & 233 & 228 & 68.115 & 31.8849 \\
H2 & 208 & 19.5177 & 255 & 233 & 228 & 82.2222 & 17.7778 \\
& 209 & 20.9228 & 255 & 233 & 228 & 82.9410 & 17.0589 \\
MH2 & 209 & 20.9228 & 255 & 233 & 228 & 83.2118 & 16.7882 \\
& 208 & 20.4545 & 255 & 233 & 228 & 82.7917 & 17.2083 \\
H3 & 218 & 15.5455 & 255 & 233 & 228 & 80.2434 & 19.7565 \\
& 218 & 15.4646 & 255 & 233 & 228 & 80.9263 & 19.0736 \\
MH3 & 218 & 15.0689 & 255 & 233 & 228 & 81.0021 & 18.9978 \\
\hline
\end{tabular}




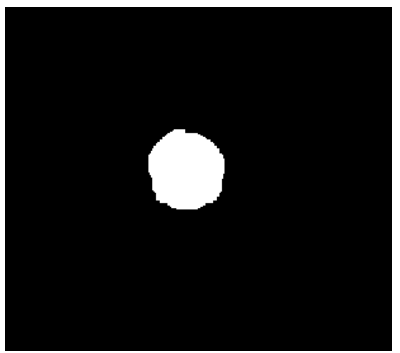

(a)

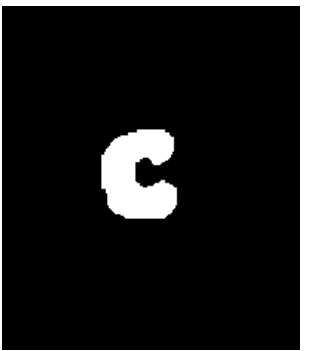

(b)

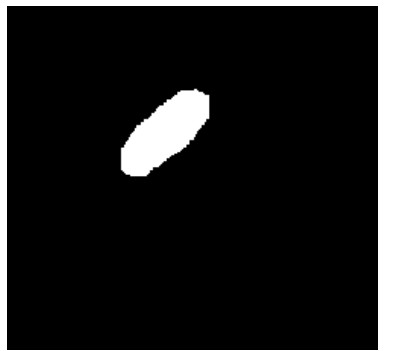

(c)

Figure 10. (a) Segmentation of a circular red blood cell; (b) Segmentation of a sickle red blood cell; (c) Segmentation of an elliptical red blood cell.

The tables are divided into two groups for each segmented red blood cell. In a first table are morphological descriptors and in a second color descriptors. In each table the horizontal side contains the following descriptors: area: represents the number of pixels lying on the whole surface of the segmented cell (white part in Figure 10(a) and Figure 10(c) above), the perimeter which is the sum of the pixels is lying on the contour of the segmented cell, the compactness, eccentricity and convexity of the cell (red blood cell). In the second table we find the average intensity (intMoy) of the pixels, the standard deviation, the average color (ColMR, ColMG, colMB) in each RGB component and the percentages of the white (\% white) of the center of the red blood cells and the colored part At the vertical level, capXXX indicates the identifier of each acquired image, $H\{1,2$, $3, \ldots, \mathrm{n}\}$ represents the set of segmented red blood cells and $\boldsymbol{M H}\{1,2,3, \ldots, \mathrm{n}\}$ indicates the average of the measurements made on each red blood cell. On healthy patients morphological and colorimetric measurements were made on the red cells of the images acquired. It can be seen that the red blood cells are circular, which is justified by their compactness values varying between 0.9 and 1. Healthy red blood cells are practically the same size in view of their perimeters which is around 272 as indicated in Table 4 above. The color of the healthy red blood cells is practically red this is noticed by the average value calculated in each channel of the RGB space $(\mathrm{R}=250, \mathrm{G}=224$ and $\mathrm{B}=220)$. This observation is confirmed by the low standard deviation $(S T D)$ whose value is around 5 which reflects the homogeneity of the red blood cell. This density of the red color is justified by its high proportion which varies from $84 \%$ to $94 \%$ this observation is made through the data in Table 5 above.

Table 6 above contains the morphological parameters of annulocytes. We find that the parameters are virtually identical to those in Table 4 above see compactness, eccentricity, and the Pbinary convexity variable. So, in order to precisely identify the annulocytes we associate with the morphological parameters the colorimetric parameters of Table 7 above because without the color aspect it could be confusing. In terms of colorimetric parameters, we find a low density of the red color which is around $61 \%$ which is lower than the values of the healthy red blood cells of Table 5 ( $84 \%$ to $94 \%$ ). The white zone in an annulocyte (39\% of the area of the red blood cell) is almost 8 times the white area of a healthy red 
blood cell ( $5 \%$ of the area of the red blood cell). These values were obtained by semi-automatic contour segmentation to calculate the area of the white area inside the cell (Section 2.4.2 d-c).

In sickle cell patients red blood cells are sickle-shaped cells or banana cells. Like all acquired images we have made morphological measurements grouped in Table 8 above. At the analysis we find that the cells do not circular in view of the value of their compactness which vary between 0.4 and 0.6 . They are also elongated and this is justified by their values of eccentricity ranging from 0.7 to 0.8 but this descriptor is not efficient enough because in our work we have also found that the elongated cells called elliptocytes have the same eccentricities as the sickle cells observation made in Table 10 above. For a more precise identification we used the convex set marked by the value of the variable PBINARY. This value is 1 if the object is non-convex and 0 if the object is convex. When we associate compactness with the study of the convex set of sickle cell cells, our system makes it possible to clearly identify the sickle cell. The coloring of the sickle cells is substantially red, which is $\mathrm{H1}$ : first red blood cell, $M H 1$ : mean red blood cell measurements H1, INTMOY average pixel intensity, colMR, CoIMG, ColMB, average staining of $R G B$ components, STD: standard deviation,\% COLOR and\% WHITE, percentage of the colored part and the white part of the red blood cells.

Elliptocytes are elongated red blood cells with rounded ends. Figure 9 above that is justified by their compactness values varying between 0.7 and 0.9 . Their elongated shape is also justified by their eccentricity values which vary between 0.7 and 0.9 (eccentricity less than 1). The identification of this cell form will be done with the combination of its compactness and eccentricity which are two descriptors discriminating for this form this analysis is made through the data measured in Table 10 above. The colorimetric parameters related to this form in Table 11 above shows that some elliptocytes have clear central zones that are more developed than others. Erythrocyte1 $(H 1)$ has $31 \%$ white and $69 \%$ colored and erythrocyte $2(H 2)$ has $17 \%$ white and $83 \%$ white. The instability of the coloration shows that the color is not a discriminating factor for this form. Then only the morphological parameters will lead us to a good identification of the elliptic shapes.

H1: first red blood cell, MH1: mean red blood cell measurements H1, intMoy: average pixel intensity, $\operatorname{colMR}, \operatorname{ColMG}, \operatorname{ColMB}$, average staining of RGB components, STD: standard deviation, \% COLOR and \% WHITE: percentage of the colored part and the white part of the red blood cells.

\section{Conclusion}

In this paper, we propose a new instrumentation approach to automatically characterize different anemia. The work done in this document consisted mainly of elaborating patient eligibility criteria for our study. Then we collected our samples and prepared the blood smears. The acquisition phase took place in an en- 
vironment described in this paper. For a better identification of each of the forms of red blood cells specific to anemia types, we propose efficient and specific descriptors for both the geometric shapes and the color appearance of red blood cells. Thus, for a formal identification of shapes, we associated the geometric descriptors (area, perimeter, compactness, eccentricity, the convex set) with the colorimetric descriptors (mean pixel intensity, standard deviation, average color for each RGB component, percentage color and white). This combination of parameters allows good identification. The extraction of these morphological and colorimetric characteristics of normal and abnormal red blood cells was possible thanks to our semi-supervised contour selection segmentation method and to the algorithms we developed and implemented under Matlab 2016a. Our future work will concern the further identification of certain forms of red blood cells and will end with the classification of anemias based on the morphology and color of red blood cells.

\section{Conflicts of Interest}

The authors declare no conflicts of interest regarding the publication of this paper.

\section{References}

[1] WHO (1993-2005) Worldwide Prévalence of Anémia, Global Data Base on Anémia. http://www.who.int/

[2] Africa Check (2018) fr.africacheck.org/Reports, Posted: July 12, 2018.

[3] WHO (2014) Global Nutrition Targets 2025: Anaemia Policy Brief (WHO/NMH/ NHD/14.4). World HealthOrganization, Geneva.

[4] Berge, F., Maggiore, G. and Frenkian, A. (2012) Update on Technological Advances in Hemocytometry at JIB 2011; IRBM NEWS.

[5] Lewis, S.M., Bain, B.J. and Bates, I. (2011) Dacie and Lewis Practical Haematology, 11th Edition, Churchill Livingston Elsevier, Edinburgh.

[6] Fossat, C. and Roméo, M. (2006) Schizocytes: Research and Interpretation. Spectra Biologie No. 150, January-February-March 2006.

[7] Geneviève, F., Galoisys, A.G., Bataille, D.M., Wanger-Ballon, O., Trimoreau, F., Fenneteau, O. and Schillinger, F. (2014) Microscopic Review of the Blood Smear: Proposals of the French-Speaking Group of Cellular Haematology (GFHC). Hematology Blood Smear Review, 156, No. 317.

[8] Zini, G., d’Onofrio, G., Briggs, C., Erber, W., Jou, J.M., Lee, S.H., McFadden, S., Vives-Corrons, J.L., Yutaka, N. and Lesesve, J.F. (2012) ICSH Recommendations for Identification, Diagnostic Value, and Quantitation of Schistocytes. International Journal of Laboratory Hematology, 34, 107-116. https://doi.org/10.1111/j.1751-553X.2011.01380.x

[9] Elsalamony, A. (2017) Anaemia Cells Detection Based on Shape Signature Using Neural Networks. Measurement, 104, 50-59. https://doi.org/10.1016/j.measurement.2017.03.012

[10] Chetaoui, C., Djemal, K., Djouak, A. and Maarff, H. (2005) Study of Forms of Sickle Cell Globules by Digital Processing of Images. 3rd International Conference. Sciences of Electronic, Technologies of Information and Telecommunications, Tu- 
nisia, 27-31 March 2005.

[11] Frejilichowski, D. (2011) Identification of Erythrocyte Types in Greyscale MGG Images for Computer-Assisted Diagnosis. IbPRIA, Lecture Notes in Computer Science, 6669, 636-643. https://doi.org/10.1007/978-3-642-21257-4_79

[12] Tomaria, R., Wan Zakaria, W.N., Abdul Jamil, M.M., Mohd Nor, F. and Nik Fuad, N.F. (2014) Computer Aided System for Red Blood Cell Classification in Blood Smear Image. Procedia Computer Science, 42, 206-213. https://doi.org/10.1016/j.procs.2014.11.053

[13] Das, D.K., Chakraborty, C., Mitra, B., Maiti, A.K. and Ray, A.K. (2012) Quantitative Microscopy Approach for Shape-Based Erythrocytes Characterization in Anemia. Journal of Microscopy, 249, 136-149. https://doi.org/10.1111/jmi.12002

[14] WHO (2014) Basic Technique for Microscopic Diagnosis of Malaria. Trainee Guide 2nd Edition, World HealthOrganization, Geneva.

[15] Abderrahim, A. and Benbabouche, F. (2014) Analyse des images cellulaires: Segmentation et caractérisation. Abu Bakr Belkaïd University, Tlemcen.

[16] Shivhare, P. and Gupta, V. (2015) Review of Image Segmentation Techniques Including Pre \& Post Processing Operations. International Journal of Engineering and Advanced Technology, 4, Issue 3.

[17] Thakur, A.K. and Madhusudan (2016) Image Segmentation: A Comparative Study. International Journal of Advances in Computer Science and Technology, 5, 64-71. http://www.warse.org/IJACST/static/pdf/file/ijacst01562016.pdf

[18] Zheng, X., Wang, Y., Wang, G.Y. and Liu, J.G. (2018) Fast and Robust Segmentation of White Blood Cell Images by Self-Supervised Learning. Micron, 107, 55-71. https://doi.org/10.1016/j.micron.2018.01.010

[19] Benomar, M.L. (2018) Combinaison adaptative des informations texture et couleur pour la segmentation d'images médicales. Universite Abou-Bekr Belkaid, Tlemcen, Laboratoire Génie Biomédical (GBM) BP 119, Tlemcen.

[20] Belaroussi, R. (2012) Image and Video Processing with Practical Exercises in Matlab and $\mathrm{C}++$. Ed Ellipses, 122-123.

[21] Jacques, B. (2001) The Biological Analysis Technician: Theoretical and Practical Guide. EMint: Editions Medicales International, Editions TEC \& DOC, Tours, 607-609. 